\title{
Piezocomposites metal-cerámica como elementos activos en acelerómetros
}

\author{
P. OCHOA ${ }^{1}$, M. VILLEGAS', J.L. PONS ${ }^{2}$, M.A. BENGOCHEA ${ }^{3}$, J.F. FERNÁNDEZ ${ }^{1}$ \\ 'Departamento de Electrocerámica, Instituto de Cerámica y Vidrio, CSIC. 28500 Arganda del Rey, Madrid \\ ${ }^{2}$ Instituto de Automática Industrial, CSIC, 28500 Arganda del Rey Madrid \\ ${ }^{3}$ KERABEN S.A., 12520 Nules, Castellón
}

\begin{abstract}
Los materiales cerámicos piezoeléctricos presentan un número importante y en continuo aumento de aplicaciones. Entre éstas su utilización como elementos activos en el diseño de acelerómetros posibilita el control de vibraciones en la industria del transporte, aerospacial, robótica e ingeniería civil entre otras. Entre los múltiples diseños como material compuesto destaca, gracias a sus elevados coeficientes de carga efectivos, el denominado tipo címbalo. En este trabajo se comprueba que modificando la geometría y el tamaño de las cápsulas se optimiza el comportamiento del sensor. Concretamente una disminución en el tamaño de las cápsulas se traduce en un aumento de la sensibilidad del acelerómetro. También se muestran los espectros de impedancia y se comparan distintas propiedades como son la frecuencia de resonancia, coeficiente de acoplo electromecánico y tiempo mínimo de respuesta.
\end{abstract}

Palabras clave: Acelerómetro, sensor de impacto, piezoeléctrico, címbalo

Piezoelectric materials as driving element in accelerometers

Piezoelectric ceramic materials have an important number of applications that is continuing increasing. One of these applications is as driving element in the design of an accelerometer. They may be used in monitoring vibration for the automobile and aeroespacial industries and robotic or civil engineering. The cymbal transducer is a good candidate for accelerometer applications due to its high effective piezoelectric charge coefficients. In this work it is shown that changes in the geometry and size of the caps improve the performance of the sensor. The sensitivity of the cymbal accelerometer increases with decreasing cap size. The mechanical vibration response of the cymbal is studied and different properties, like resonant frequency, electromechanical coupling coefficient, and fastest response time are compared.

Keywords: Accelerometer, impact sensor, piezoelectric, cymbal

\section{INTRODUCCIÓN}

El coste de producción de los acelerómetros ha disminuido drásticamente en los últimos años, haciendo posible que muchas aplicaciones en las que el precio es un factor importante sean ahora económicamente viables. Por ello los acelerómetros se encuentran, cada vez más, en productos comerciales y de consumo.

El principio de funcionamiento de un acelerómetro se basa en la ley de Hook y la segunda ley de Newton. La aceleración genera una fuerza sobre una masa, denominada masa sísmica, que al desplazarse deforma un muelle (1) con la condición de que:

$$
F=m a=k x
$$

De esta forma una aceleración $a$ ocasionará que la masa se desplace, o viceversa. Si se observa un desplazamiento $x$ suponemos que la masa está bajo la acción de una aceleración:

$$
a=\frac{k x}{m}
$$

Hay que señalar que este sistema solo responde a aceleraciones a lo largo de la dirección del muelle. Es lo que se denomina un acelerómetro de un solo eje. Para conseguir medidas de aceleración en múltiples ejes el sistema debe ser duplicado a lo largo de cada uno de los ejes requeridos.
No importa cual sea el diseño del sensor o cual se la técnica de conversión, el objetivo final de la medida es la detección del desplazamiento de la masa, denominada masa sísmica, respecto a la carcasa. De este modo cualquier sensor de desplazamiento capaz de medir desplazamientos microscópicos bajo fuertes vibraciones puede ser empleado como acelerómetro. Los tipos de acelerómetros más comunes son: capacitivos, piezorresistivos, servo acelerómetros, de fibra óptica y piezoeléctricos.

En el caso de los acelerómetros piezoeléctricos, cuando la masa sísmica se desplaza por efecto de la aceleración, ejerce una fuerza sobre el cristal piezoeléctrico y en su interior se produce una redistribución de las cargas generándose una diferencia de carga neta entre sus caras que es proporcional a la aceleración.

Un acelerómetro se puede emplear para medir velocidades, desplazamientos o fuerzas. Algunas aplicaciones de medida de fuerzas inerciales incluyen sensores de choque para airbags, sistemas de navegación para coches y controladores de ascensores. Como detectores de impacto se emplean en aplicaciones tales como control de envíos o comprobación de caídas (2). En la industria se pueden aplicar en control de vibraciones, detección de inclinaciones, estabilización de plataformas, realización de análisis modales y control del estado 
de funcionamiento de la maquinaria (3). En automoción las aplicaciones son múltiples: sistemas electrónicos de control de la suspensión, pruebas de vibración y choque, control dinámico de vehículos (medición de los movimientos en todas las direcciones) y administración de la flota comercial (4). Los ingenieros están instrumentando progresivamente estructuras para la detección de fatiga, resonancia y respuesta bajo carga (5), en estas aplicaciones, los acelerómetros son empleados para análisis modales, mantenimiento preventivo y control remoto de estructuras tales como tuberías, torres de radio, puentes, edificios, etc. Y en medicina se emplean para controlar los movimientos del corazón en dispositivos médicos implantables (6).

\section{ACELERÓMETROS PIEZOELÉCTRICOS}

El efecto piezoeléctrico consiste en la polarización eléctrica que tiene lugar en ciertos cristales, carentes de centro de simetría, cuando son sometidos a acciones mecánicas aplicadas según direcciones cristalinas determinadas (1).

En un acelerómetro, la tensión aparece como resultado de la fuerza que ejerce la masa sísmica sobre el cristal piezoeléctrico, que actúa como muelle (7). De esta forma, la cantidad total de carga acumulada es proporcional a la fuerza aplicada, y la fuerza aplicada es proporcional a la aceleración. Los electrodos y cables conectados transmiten la carga a un acondicionador de señal que puede ser remoto o integrado en el acelerómetro. Una vez que la señal es acondicionada puede ser grabada, analizada o monitorizada.

Existen varias configuraciones mecánicas que se definen según sea la naturaleza de la fuerza inercial que la masa acelerada ejerce sobre el material piezoeléctrico, las tres básicas son: compresión, flexión o cortante (8).

Independientemente del modo de trabajo empleado, la sensibilidad del acelerómetro dependerá de los coeficientes piezoeléctricos del material.

Las ventajas de los acelerómetros piezoeléctricos frente a otros diseños son: circuitos menos complicados, capacidad de soportar mayores niveles de impacto, y el funcionamiento con bajo consumo. Los diseños piezoeléctricos a pesar de no poder medir aceleraciones estáticas, pueden ser diseñados para medir frecuencias tan bajas como $0.1 \mathrm{~Hz}$. Estos acelerómetros ofrecen también frecuencias de resonancia extremadamente altas en comparación con otros tipos de acelerómetro.

\section{ACELERÓMETROS PIEZOELÉCTRICOS BASADOS EN DISEÑOS TIPO CÍMBALO}

El diseño tipo címbalo consiste en un disco piezoeléctrico encapsulado entre dos cápsulas metálicas con una cavidad en forma de cono truncado (10). Estas cápsulas tienen forma de platillo de orquesta o címbalo, de ahí su nombre (figura 1). Las cápsulas actúan transformando una fuerza perpendicular a una de ellas en dos componentes, una transversal y otra radial, de forma que tanto el coeficiente piezoeléctrico $d_{33}$ como el $\mathrm{d}_{31}\left(=\mathrm{d}_{32}\right.$ para discos $)$ contribuyan a la respuesta del sensor (11). Esto provoca que el coeficiente de carga efectivo, $\mathrm{d}_{33}$ eff, sea muy superior al $\mathrm{d}_{33}$ del material.

Un diseño alternativo de las cápsulas, con una forma similar a un dedal, en el que se reduce el tamaño y se elimina la corona plana exterior (figura 1) ha demostrado buenos resultados (12).
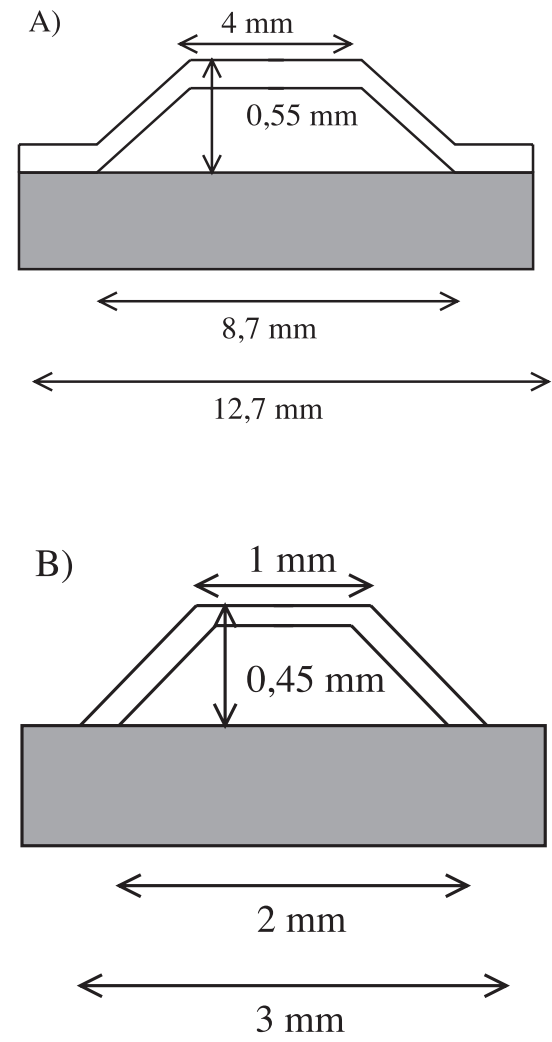

C)

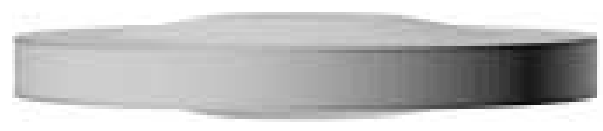

D)

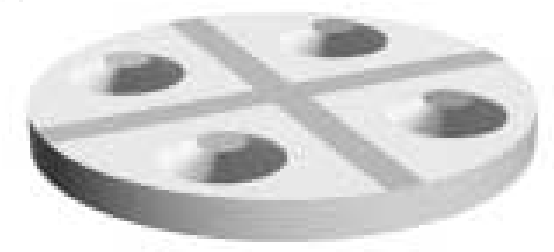

Figura 1: a) Cápsula tipo címbalo de 12,7 mm de diámetro. b) Cápsulas tipo címbalo modificado de $3 \mathrm{~mm}$ de diámetro. c) Címbalo simétrico con cápsulas de $12.7 \mathrm{~mm}$. de diámetro. d) Cuatro címbalos modificados de $3 \mathrm{~mm}$ de diámetro sobre un disco electrodado en cuartos.

Una medida de la cantidad de energía eléctrica convertida en energía mecánica es el factor de acoplo electromecánico, $\mathrm{k}_{\text {eff' }}$ y su valor máximo es 1. Puede ser calculado a partir de la frecuencia de resonancia, $\mathrm{f}_{\mathrm{r}^{\prime}} \mathrm{y}$ la frecuencia de antiresonancia, $\mathrm{f}_{a^{\prime}}$, según la ecuación:

$$
k_{e f f}^{2}=\frac{f_{a}^{2}-f_{r}^{2}}{f_{a}^{2}}
$$


También, a partir de la frecuencia de resonancia, $\mathrm{f}_{\mathrm{r}^{\prime}}$ el tiempo mínimo de respuesta, $\mathrm{t}_{\text {res }^{\prime}}$ puede ser calculado aplicando la ecuación :

$$
t_{\text {res }}=\frac{1}{f_{r}}
$$

En un címbalo la fuerza ejercida por la una masa (m) es transferida por la aceleración, $\mathrm{F}=\mathrm{ma}$, a través de las cápsulas metálicas al disco cerámico (9). La carga generada por el címbalo será:

$$
\mathrm{Q}=\mathrm{d}_{33}{ }^{\text {eff }} \mathrm{F}
$$

donde $Q$ es la carga generada, $F$ la fuerza ejercida $\mathrm{y} \mathrm{d}_{33}{ }^{\text {eff }}$ el coeficiente piezoeléctrico efectivo.

Una magnitud importante para caracterizar un acelerómetro es la sensibilidad del mismo. Se define la sensibilidad de carga, $\mathrm{S}_{\mathrm{q}^{\prime}}$ como la carga generada por unidad de aceleración (7):

$$
S_{q}=\frac{Q}{a}=d_{33}^{e f f} m \quad[p C / g]
$$

También se puede definir la sensibilidad de tensión, $S_{V^{\prime}}$ como el voltaje por unidad de aceleración [7]. Podemos relacionar ambos factores de sensibilidad teniendo en cuenta que si C es la capacidad del condensador formado por el disco piezoeléctrico al metalizar las caras superior e inferior, el voltaje será $\mathrm{V}=\mathrm{q} / \mathrm{C}$, y dado que la capacidad es a su vez, $\mathrm{C}=\varepsilon \mathrm{A} / \mathrm{t}$, entonces:

$$
S_{V}=\frac{V}{a}=\frac{t}{\varepsilon \cdot A} S_{q}=\frac{t \cdot m}{\varepsilon \cdot A} d_{33}^{e f f} \quad[\mathrm{mV} / \mathrm{g}]
$$

siendo A el área, t el espesor y $\varepsilon$ la permitividad dieléctrica del disco.

Una aplicación particular de un acelerómetro es la medida de impactos. En estos un objeto sufre un cambio brusco en su velocidad en un intervalo de tiempo muy corto. Los datos deben expresarse en unidades de sensibilidad (voltaje o carga generada por unidad de aceleración), y, dado que el sistema de medida da los resultados en voltios, se debe calcular la aceleración con que se produce el impacto. Se puede calcular la velocidad con que la masa llega al címbalo a partir de las leyes de conservación de la energía:

$$
m g h=\frac{1}{2} m v^{2}
$$

siendo $\mathrm{m}$ la masa de la pesa, $\mathrm{h}$ la altura desde la que se deja caer, $\mathrm{g}$ la aceleración de la gravedad y v la velocidad final. En el momento del impacto la masa varía su velocidad $\mathrm{v}$ hasta detenerse, por lo tanto, la aceleración promedio durante el impacto es, aproximadamente, $\mathrm{v}$ dividida por el tiempo de duración del impacto. Este tiempo de duración del impacto se puede estimar a partir de la anchura del pico de voltaje en el osciloscopio.

\section{PROCEDIMIENTO EXPERIMENTAL}

Los sensores se fabricaron a partir de discos cerámicos, PZT 5A, electrodados (12.7 mm de diámetro y $1.0 \mathrm{~mm}$ de espesor) y cápsulas metálicas de Kovar (aleación Fe, Ni, Co). En el diseño de las cápsulas se emplearon dos geometrías diferentes, las primera con $12.7 \mathrm{~mm}$ de diámetro y un diseño tipo címbalo. El sensor, en el caso de tener una sola cápsula, se denomina címbalo asimétrico, y si son dos cápsulas, címbalo simétrico. La segunda geometría es de $3 \mathrm{~mm}$ de diáme- tro y tiene el nuevo diseño tipo "dedal", sin la corona exterior del címbalo. En el caso de estas cápsulas con el nuevo diseño los discos cerámicos se electrodaron en cuartos, con una cápsula en cada uno, para obtener una señal independiente de cada una de ellas. Los discos cerámicos y las cápsulas metálicas se unieron mediante una resina epoxy bicomponente, teniendo especial cuidado en no rellenar la cavidad. Con el fin de estudiar la respuesta fue necesaria la adicción de una masa externa o masa inercial, que en todos los casos fue de 1,2 g.

Para la realización de las medidas de sensibilidad se ha empleado un actuador electromagnético gobernado por un generador de funciones (Hewlett Packard 33120 A) sobre el que se ha programado un barrido de ondas tipo senoidal en el intervalo de frecuencias de interés $(0-1000 \mathrm{~Hz})$. Dado que en el sistema únicamente ejerce control sobre la tensión de alimentación que el generador aporta al actuador, se ha utilizado un acelerómetro comercial (B\&K 4375) para calibrar el sistema. A través de la medida del acelerómetro comercial se ha obtenido la amplitud de la aceleración excitada a cada frecuencia.

Se determinó la sensibilidad en impacto mediante un ensayo en el que se mide el voltaje generado por un címbalo sometido al impacto de una masa de 16,18 $\pm 0,01 \mathrm{~g}$ desde una altura de 3,0 $\pm 0,1 \mathrm{~cm}$. La señal generada se amplifica mediante un amplificador de voltaje diseñado en el Departamento de Electrocerámica del ICV, CSIC, para la realización de estas medidas. Una vez amplificada la señal se controla con un osciloscopio (Hitachi, V-550), midiéndose la altura y la anchura del pico de voltaje en la pantalla del mismo, que fue de 0,8 $\pm 0,1 \mathrm{~ms}$ en todos los casos. A partir de estos datos se calculó la aceleración del impacto, $98 \pm 7 \mathrm{~g}\left(1 \mathrm{~g}=9,8 \mathrm{~m} / \mathrm{s}^{2}\right)$.

Para el cálculo del coeficiente de acoplo electromecánico y de la velocidad de respuesta se midieron las frecuencias de resonancia y antiresonancia de los distintos diseños a partir de los espectros de impedancia obtenidos con un analizador de impedancia (Hewlett Packard, Model 4192A LF) acoplado a un ordenador personal (Hewlett Packard,Vectra 286/12). Las medidas se realizaron con una resolución de $100 \mathrm{~Hz}$ y un nivel de oscilación de $0.2 \mathrm{~V}$ para evitar fenómenos de polarización.

\section{RESULTADOS Y DISCUSIÓN}

En la tabla I se muestran los valores de la sensibilidad de voltaje como acelerómetro para los distintos diseños de címbalos. Estos resultados se han obtenido sin necesidad de amplificación.

TABLA I: SENSIBILIDAD DE VOLTAJE COMO ACELERÓMETRO Y COMO SENSOR DE IMPACTO PARA DISTINTOS DISEÑOS DE CÍMBALO

\begin{tabular}{|c|c|c|}
\hline & $\begin{array}{c}S_{V} \text { acelerómetro } \\
( \pm 10 \mathrm{mV} / \mathrm{g})\end{array}$ & $\begin{array}{c}S_{\mathrm{V}} \text { impacto } \\
( \pm 2 \mathrm{mV} / \mathrm{g})\end{array}$ \\
\hline $\begin{array}{c}\text { Címbalo Simétrico } \\
\mathrm{d}=12,7 \mathrm{~mm}\end{array}$ & 350 & 36 \\
\hline $\begin{array}{c}\text { Címbalo Asimétrico } \\
\mathrm{d}=12,7 \mathrm{~mm}\end{array}$ & 240 & 7 \\
\hline $\begin{array}{c}\text { Címbalo Modificado } \\
\mathrm{d}=3 \mathrm{~mm}\end{array}$ & 1.000 & 20 \\
\hline
\end{tabular}


Al reducir el tamaño de la cavidad y cambiar la geometría de la cápsula, reduciendo la superficie de unión con el disco, se aumenta la sensibilidad como acelerómetro desde 240-350 $\mathrm{mV} / \mathrm{g}$ para címbalos de $12,7 \mathrm{~mm}$, hasta $1000 \mathrm{mV} / \mathrm{g}$ para los címbalos modificados, tipo dedal, de $3 \mathrm{~mm}$.

Los acelerómetros comerciales (13) tienen sensibilidades de voltaje de $10 \mathrm{mV} / \mathrm{g}$, y los denominados de "alta sensibilidad" alcanzan valores desde 100 hasta $300 \mathrm{mV} / \mathrm{g}$. Si se compara con los valores obtenidos con los diseños de sensores tipo címbalo se ve que son iguales, en el caso de los címbalos simétrico y asimétrico de $12,7 \mathrm{~mm}$, o claramente superiores en el caso del címbalo modificado de $3 \mathrm{~mm}$ de diámetro. Aún así esta comparación no es equitativa pues los acelerómetros comerciales llevan incorporados circuitos integrados que actúan como amplificadores de carga. Es decir, incluso en el peor de los casos se obtienen sensibilidades comparables a las de los dispositivos comerciales, pero sin necesidad de amplificación. Esto trae consigo dos ventajas muy importantes, por un lado su menor tamaño, y por otro, debido a que pueden operar sin amplificador de carga, se puede eliminar la fuente de alimentación externa para su funcionamiento.

Los valores de la sensibilidad como sensor de impacto se muestran en la tabla II. La respuesta a impacto de los piezocomposites no es tan espectacular como su respuesta como acelerómetro, pero aún así son comparables a las respuestas de los sensores comerciales (13), pero sin necesidad de amplificación. Esto supone, como ya se había mencionado anteriormente, una notable reducción en el tamaño y la ausencia de fuentes de alimentación externas.

En este caso la reducción de tamaño y el cambio de geometría en la cápsula de los címbalos también significa un aumento de la sensibilidad, los címbalos de $3 \mathrm{~mm}$ casi triplican en sensibilidad a los címbalos asimétricos de 12,7 mm. Los címbalos simétricos de $12,7 \mathrm{~mm}$ sí presentan mejores resultados que los de $3 \mathrm{~mm}$, pero tienen el problema de ser más difícil su integración.

Al realizar los ensayos de impacto se debe tener en cuenta que el disco cerámico es frágil, y un impacto de las características del ensayado significa la rotura del mismo. La cápsula metálica actúa como un muelle, absorbiendo la mayor parte del choque y aumentando así la vida útil del sensor, al ser este capaz de soportar un mayor número de impactos. Mientras que en un disco cerámico se produce la rotura al primer impacto los diseños ensayados soportaron más de doce ensayos sin que aparecieran fisuras. Este efecto es menos acusado en los címbalos modificados de $3 \mathrm{~mm}$ de diámetro que ofrecen una menor protección al elemento cerámico frente a impacto, aparecen grietas al quinto ensayo, si bien es un punto interesante de abordar en investigaciones futuras.

Los espectros de impedancia en función de la frecuencia obtenidos para los címbalos de $12.7 \mathrm{~mm}$ de diámetro, simétrico y asimétrico, y para los címbalos modificados tipo dedal de $3 \mathrm{~mm}$ se muestran en las figuras 2 y 3 respectivamente.

En el espectro de impedancia del címbalo simétrico (figura 2) se identifican dos modos fundamentales de vibración, a $34 \pm$ $1 \mathrm{kHz}$ el pico (doble en el $80 \%$ de los casos) debido a la vibración de las cápsulas metálicas, y a mayores frecuencias el modo radial de vibración del disco piezoeléctrico cerámico. La razón del desdoblamiento de la frecuencia de resonancia del modo de la cavidad se encuentra en la asimetría de los diferentes parámetros de las cápsulas: la altura, el diámetro de la cavidad, la unión metal cerámica y la existencia de deformaciones asimétricas (14). Se asume por lo tanto que cada frecuencia de resonancia corresponde a una de las cápsulas metálicas.
TAbla II: Propiedades de loS DifERENTES DiseÑOS DE SENSOR

\begin{tabular}{|c|c|c|c|}
\hline & $\begin{array}{c}\text { Frecuencia de } \\
\text { resonancia } \\
( \pm 1 \mathrm{kHz})\end{array}$ & $\begin{array}{c}\text { Tiempo mínimo } \\
\text { de respuesta } \\
( \pm 0,1 \mu \mathrm{s})\end{array}$ & $\mathrm{k}_{\text {eff }}( \pm 0,1 \%)$ \\
\hline $\begin{array}{c}\text { Címbalo Asimétrico } \\
\mathrm{d}=12,7 \mathrm{~mm}\end{array}$ & 32 & 31,2 & 1,7 \\
\hline Címbalo Simétrico d=12,7mm & 34 & 29,4 & 3,2 \\
\hline Címbalo Modificado d=3 mm & 144 & 6,9 & 1,5 \\
\hline
\end{tabular}

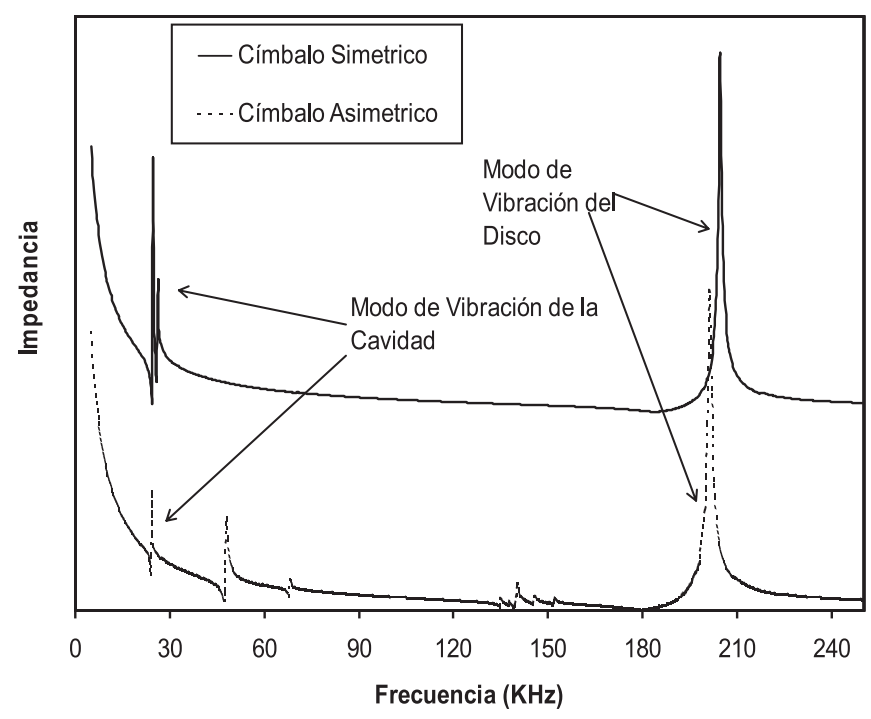

Figura 2: Espectro de impedancia en función de la frecuencia de címbalos simétricos y asimétricos

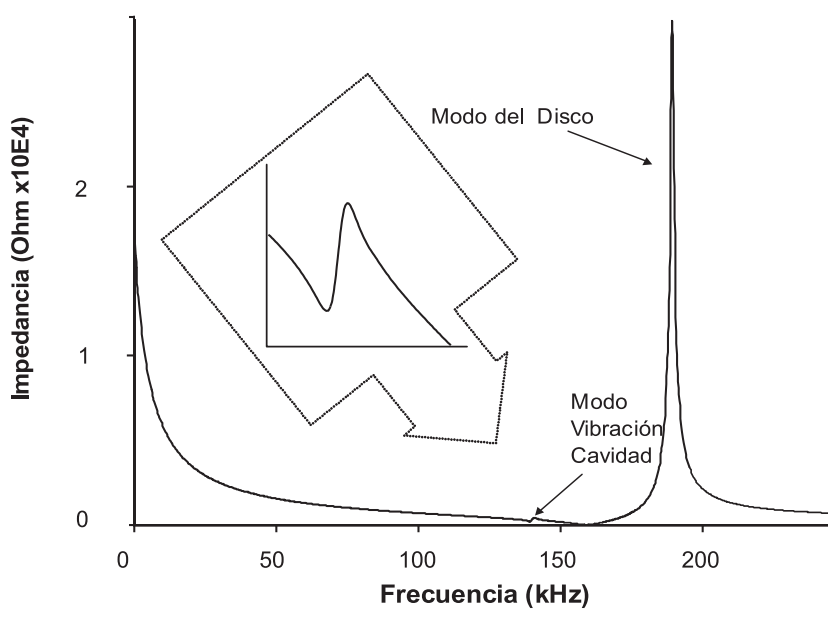

Figura 3: Espectro de impedancia de un címbalo modificado tipo dedal de $3 \mathrm{~mm}$ de diámetro 
En los címbalos asimétricos (figura 2) el primer pico del espectro de impedancia se identifica con el modo de resonancia de la cavidad, los picos en la región entre el modo de la cavidad y el del disco cerámico no aparecen en el espectro del címbalo simétrico, por lo que es razonable asociarlos a modos de flexión de la estructura, que estarían impedidos en el caso de tener dos cápsulas metálicas.

Al estudiar el espectro de impedancia del disco con un címbalo modificado tipo dedal de $3 \mathrm{~mm}$ de diámetro (figura 3) solo se observan dos picos, el modo radial del disco a una frecuencia de $160 \pm 1 \mathrm{kHz}$, en la misma posición que el disco cerámico sin encapsular, y otro modo a $144 \pm 1 \mathrm{kHz}$ que es el modo de resonancia de la cavidad.

En la tabla II se recogen los resultados obtenidos en la medida de la frecuencia de resonancia, $\mathrm{f}_{\mathrm{r}^{\prime}}$ del coeficiente de acoplo electromecánico, $\mathrm{k}_{\text {eff' }} \mathrm{y}$ del tiempo mínimo de respuesta, $t_{\text {res }}$.

Con el nuevo diseño tipo dedal destaca el aumento de la frecuencia de resonancia desde $32 \mathrm{kHz}$ hasta $144 \mathrm{kHz}$, lo que significa un aumento del intervalo de frecuencias en que puede emplearse como actuador no resonante y en el que el sensor es capaz de "seguir" la señal de excitación, es decir, el intervalo de frecuencias de trabajo.

Los factores de acoplamiento electromecánico, $\mathrm{k}_{\text {eff' }}$ son similares en el caso de los címbalos asimétricos de $12,7 \mathrm{~mm}$ y en el de los címbalos modificados tipo dedal de $3 \mathrm{~mm}, 1,6 \pm$ $0,1 \%$.

El tiempo mínimo de respuesta, es decir, la mayor velocidad de reacción, se obtiene para el címbalo modificado tipo dedal, 6,9 $\pm 0,1 \mu \mathrm{s}$. Al reducir el tamaño de los címbalos y modificar su geometría se ha disminuido el tiempo mínimo de respuesta y aumentado el intervalo de frecuencias de trabajo, manteniendo un $\mathrm{k}_{\text {eff }}$ comparable al del címbalo asimétrico de mayor tamaño.

\section{CONCLUSIONES}

Con los diseños de acelerómetros basados en címbalos se ha conseguido un aumento de la sensibilidad, sin necesidad de amplificadores de carga y, por lo tanto, sin fuentes de alimentación externas, lo que se manifiesta en una disminución del tamaño y del coste de producción a la vez que se hacen más transportables e integrables.

Se ha variado su geometría, siendo el cambio más significativo la disminución de la superficie de adhesión con el disco cerámico en proporción al diámetro total. La reducción en el tamaño desde $12,7 \mathrm{~mm}$ a $3 \mathrm{~mm}$ y el nuevo diseño de la cáp- sula metálica de estos últimos ha significado: una disminución del tiempo de respuesta y un aumento, tanto del intervalo de frecuencias de trabajo como de la sensibilidad como sensor, superior a los acelerómetros comerciales convencionales y comparable a los denominados de "alta sensibilidad", pero sin necesidad de amplificación. Por último, mantienen un factor de acoplamiento electromecánico, $\mathrm{k}_{\text {eff }}$ comparable al de címbalos asimétricos de mayor tamaño.

\section{AGRADECIMIENTOS}

Los autores agradecen el apoyo financiero para la realización de este trabajo. (EUREKA 2309 FACTORY - PAMIS y CICYT-TAP99-1244-CO2-01 (ICARO)).

\section{BIBLIOGRAFÍA}

[1] K. Uchino, "Ferroelectric Devices", Marcel Dekker Inc., 2000.

[2] R. Shear, "Piezoelectric Sensors for OEM Applications", www.endevco.com/op-oem-0299.html

[3] J.C. Robinson, J.M. Rybak, E.R. Saller, "Using Accelerometers to Monitor Complex Machinery Vibration", Sensors, June, 36-42, (1997).

[4] G.A. MacDonald, "A Review of Low Cost Accelerometers for Vehicle Dynamics", Sensors and Actuators, A21-A23, 303-307, (1990).

[5] D. Crescini, V. Ferrari, D. Marioli, E. Sardini, A. Taroni, "Vibration and Vibrating Sensor in Thick Film Technology", Machine Vibration, 4, 161-167, (1995).

[6] J.M. Sikorski, D.A. Ruben, "High Output Sensor and Accelerometer for Implantable Medical Device", United States Patent ํㅡ을 6038475 (2000).

[7] J. Fraile, P. García, "Instrumentación Aplicada a la Ingeniería" E.T.S. Ingenieros de Caminos, Canales y Puertos, Servicio de Publicaciones, Madrid (1995).

[8] M. Serridge, T.R. Licht, "Piezoelectric Accelerometer and Vibration Preamplifier Handbook", K. Larsen \& Son A/S, DK, (1987).

[9] B. Koc, A. Dogan, J.F. Fernández, R.E. Newnham, K. Uchino, "Accelerometer Application of the Modified Moonie (Cymbal) Transducer", Jpn. J. Appl. Phys. 35, 4547-4549, (1996).

[10] P. Ochoa, M. Villegas, J.F. Fernández, "Propiedades y Aplicaciones de Piezocomposites Tipo Címbalo”, Bol. Soc. Esp. Cerám. Vidr., 40, 4, 301-308 (2001).

[11] J.F. Fernández, A. Dogan, J.F. Tressler, Q. M. Zhang, R. E. Newnham, "Hollow Piezoelectric Composites", Sensor and Actuators A Physical, 51, 183-196 (1996).

[12] P. Ochoa, "Desarrollo de Células Sensoras para su Integración en Pieles Artificiales", Proyecto Fin de Carrera, Ingeniería de Materiales, Universidad Politécnica de Madrid (2000).

[13] Catálogo de productos G-500, "Smart Sensing", PCB Piezotronics Inc.,USA (1991).

[14] J.F. Tressler, R. E. Newnham, “Doubly Resonant Cymbal-Type Transducers", IEEE Transactions on Ultrasonics, Ferroelectrics and Frequency Control", 44, 55, 1175-1177, (1997).

Recibido: 31.05 .01

Aceptado: 10.12.01 